\title{
An HST parallax of the distant cataclysmic variable V1223 Sgr, its system parameters, and accretion rate ${ }^{\star}$
}

\author{
K. Beuermann ${ }^{1}$, Th. E. Harrison ${ }^{2, \star \star}$, B. E. McArthur ${ }^{3}$, G. F. Benedict ${ }^{3}$, and B. T. Gänsicke ${ }^{4}$ \\ 1 Universitäts-Sternwarte Göttingen, Geismarlandstr. 11, 37083 Göttingen, Germany \\ 2 New Mexico State University, Box 30001/MSC 4500, Las Cruces 88003, Mexico \\ e-mail: tharriso@nmsu.edu \\ 3 McDonald Observatory, University of Texas, Austin, 78712 Texas, USA \\ e-mail: mca@barney.as.utexas.edu; fritz@astro.as.utexas.edu \\ ${ }^{4}$ Department of Physics and Astronomy, University of Southampton, Highfield, Southampton SO17 1BJ, UK \\ e-mail: btg@astro.soton.ac.uk
}

Received 1 October 2003 / Accepted 10 February 2004

\begin{abstract}
Using the Hubble space Telescope Fine Guidance Sensor, we have measured the trigonometric parallax of the bright cataclysmic variable $1223 \mathrm{Sgr}$. The absolute parallax is $\pi_{\mathrm{abs}}=1.96 \pm 0.18$ mas, making V1223 Sgr the most distant CV with a well-determined trigonometric parallax. This distance, a Lutz-Kelker correction, and the previously measured extinction yield an absolute visual high-state magnitude $M_{V}=4.0 \pm 0.2$. We outline a model, which is consistent with the observed spin-down of the white dwarf and provides for much of the UV/optical emission by reverberation of X-rays. From previous X-ray and $\mathrm{UV} /$ optical data, we derive an accretion luminosity $L_{\mathrm{acc}}=(2.6 \pm 0.8) \times 10^{34} \mathrm{erg} \mathrm{s}^{-1}$, a white dwarf mass $M_{1}=0.93 \pm 0.12 M_{\odot}$, and an accretion rate $\dot{M}=(1.4 \pm 0.3) \times 10^{17} \mathrm{~g} \mathrm{~s}^{-1}$,
\end{abstract}

Key words. astrometry - stars: individual: V1223 Sgr - stars: novae, cataclysmic variables

\section{Introduction}

V1223 Sgr (4U1849-31) is one of the brightest confirmed intermediate polars, both optically and in X-rays. The only previous distance estimate of $600 \mathrm{pc}$ was based on the observed $E_{B-V}=0.15$ and a mean reddening of $0.25 \mathrm{mag} \mathrm{kpc}^{-1}$ (Bonnet-Bidaud et al. 1982). Since V1223 Sgr is not included in ground-based parallax programs, we have obtained a high-precision parallax using the Hubble Space Telescope Fine Guidance Sensor (FGS). This observation increases the number of $\mathrm{CV}$ parallaxes measured with the HST FGS to eight (Harrison et al. 1999, 2000, 2003; McArthur et al. 1999, 2001; Beuermann et al. 2003).

V1223Sgr shows modulations at the orbital period $P_{\text {orb }}=3.366 \mathrm{~h}$, the spin period of the white dwarf $P_{\text {spin }}=745.5 \mathrm{~s}$ (Osborne et al. 1985), and the beat period $P_{\text {beat }}=794.38 \mathrm{~s}$ (Steiner et al. 1981). The UV/optical spectral energy

^ Based on observations made with the NASA/ESA Hubble Space Telescope, which is operated by the Association of Universities for Research in Astronomy, Inc., under NASA contract NAS 5-26555. These observations are associated with proposal \#9230.

$\star \star$ Visiting Astronomer, Cerro Tololo Inter-American Observatory, National Optical Astronomy Observatory, which is operated by the Association of Universities for Research in Astronomy, Inc., under cooperative agreement with the National Science Foundation. distribution was interpreted by Bonnet-Bidaud et al. (1982) and Mouchet (1983) in terms of an accretion disk which reaches close to the white dwarf and should cause it to be spun up. Unexpectedly, however, the white dwarf was found to spin down (Jablonski \& Steiner 1987; van Amerongen et al. 1987) suggesting that the disk stays much further away from the white dwarf. Our accurate distance allows us to determine the luminosity of V1223 Sgr, estimate its accretion rate, and to show that a standard intermediate-polar model is consistent with the available observations.

\section{Observations, data reduction, and results}

\subsection{HST observations}

The process for deriving a parallax for a cataclysmic variable from HST FGS observations has been described in papers by McArthur et al. (2001, 1999) and Harrison et al. (1999). The process used here is nearly identical to those efforts, as well as our recent program on EX Hya (Beuermann et al. 2003). An FGS program consists of a series of observations of the target of interest, and a set of four or more reference stars located close to that target. Typically, three epochs of observations, each comprised of two or more individual HST pointings (orbits), are used to solve for the variables in the series of 
Table 1. Photometric and spectroscopic data for the V1223 Sgr reference frame.

\begin{tabular}{lccccccccc}
\hline \hline Star & $V$ & $B-V$ & $V-R$ & $V-I$ & $J-H$ & $H-K$ & $K$ & $A_{V}$ (mag) & Spectral type \\
\hline V1223 Sgr & 13.18 & 0.02 & 0.05 & 0.15 & 0.16 & 0.06 & 12.68 & $0.47^{1}$ & \\
Ref-\#1 & 12.81 & 1.80 & 1.04 & 2.24 & 1.08 & 0.14 & 8.07 & 0.81 & M 1III \\
Ref-\#2 & 12.34 & 0.59 & 0.33 & 0.66 & 0.24 & 0.11 & 10.89 & 0.41 & F 5V \\
Ref-\#3 & 13.01 & 1.16 & 0.59 & 1.13 & 0.57 & 0.12 & 10.40 & 0.40 & G 9.5III \\
Ref-\#4 & 13.04 & 1.17 & 0.60 & 1.18 & 0.70 & 0.09 & 10.28 & 0.50 & G 9.5III \\
Ref-\#5 & 13.44 & 1.40 & 0.71 & 1.34 & 0.70 & 0.15 & 10.30 & 0.84 & K 1III \\
\hline
\end{tabular}

${ }^{1}$ From $E_{B-V}=0.15$ (Bonnet-Bidaud et al. 1982).

Table 2. Astrometric data and derived spectroscopic parallaxes for the V1223 Sgr reference frame.

\begin{tabular}{|c|c|c|c|c|c|c|c|c|c|c|c|}
\hline \multirow[t]{2}{*}{ Star } & \multirow[t]{2}{*}{$\alpha_{2000}$} & \multicolumn{2}{|c|}{$P M_{\alpha}(\mathrm{mas} / \mathrm{yr})$} & \multirow[t]{2}{*}{$\delta_{2000}$} & \multicolumn{2}{|c|}{$P M_{\delta}(\mathrm{mas} / \mathrm{yr})$} & \multirow{2}{*}{$\begin{array}{c}\xi^{1} \\
\text { (mas) }\end{array}$} & \multirow{2}{*}{$\begin{array}{c}\eta \\
\text { (mas) }\end{array}$} & \multirow{2}{*}{$\begin{array}{r}\sigma_{\xi} \\
\text { (mas) }\end{array}$} & \multirow{2}{*}{$\begin{array}{c}\sigma_{\eta} \\
\text { (mas) }\end{array}$} & \multirow{2}{*}{$\begin{array}{c}\pi \\
\text { (mas }\end{array}$} \\
\hline & & UCAC2 & FGS & & UCAC2 & FGS & & & & & \\
\hline V1223 Sgr & 185502.3 & $+2.2 \pm 2.6$ & $+2.0 \pm 0.2$ & -310949.5 & $-23.7 \pm 2.9$ & $-24.6 \pm 0.2$ & -67.090 & 790.152 & 0.37 & 0.35 & \\
\hline Ref-\#1 & 185453.3 & $-8.1 \pm 3.8$ & $-9.2 \pm 0.2$ & -311021.4 & $-8.1 \pm 4.4$ & $-5.8 \pm 0.2$ & 50.571 & 809.254 & 0.45 & 0.36 & 0.33 \\
\hline Ref-\#2 & 185442.3 & $-0.2 \pm 2.6$ & $-0.5 \pm 0.2$ & -310910.1 & $-13.7 \pm 3.0$ & $-13.9 \pm 0.2$ & 183.174 & 722.876 & 0.60 & 0.49 & 1.91 \\
\hline Ref-\#3 & 185449.2 & $-7.9 \pm 3.8$ & $-5.5 \pm 0.2$ & -310759.6 & $-18.0 \pm 4.4$ & $-17.8 \pm 0.2$ & 87.710 & 662.492 & 0.42 & 0.45 & 0.40 \\
\hline Ref-\#4 & 185501.5 & $+1.3 \pm 3.8$ & $-0.7 \pm 0.2$ & -310753.2 & $-7.4 \pm 4.4$ & $-7.4 \pm 0.2$ & -70.136 & 673.396 & 0.41 & 0.45 & 0.41 \\
\hline Ref-\#5 & 185507.1 & $+3.3 \pm 3.8$ & $+4.4 \pm 0.2$ & -310955.7 & $-2.4 \pm 4.4$ & $-0.9 \pm 0.2$ & -128.303 & 803.079 & 0.49 & 0.54 & 0.37 \\
\hline
\end{tabular}

${ }^{1} \xi$ and $\eta$ are the relative positions in arcseconds from Eqs. (3) and (4), $\sigma_{\xi}$ and $\sigma_{\eta}$ their standard deviations.

equations that define a parallax solution. For V1223 Sgr, we obtained observations on four different epochs (2000 September, 2001 March, 2001 September, and 2002 September) during which nine orbits of HST time were used. The extra epoch of observation was essential in deriving the high precision in the smallest parallax we have yet measured using the FGS. Extensive calibration data, as well as estimates of the distances and proper motions of the reference stars, are required to obtain a robust parallax solution.

\subsection{Spectroscopic parallaxes of the reference frame}

We have used a combination of spectroscopy and photometry to estimate spectroscopic parallaxes for the reference stars. The optical BVRI photometry of the five reference stars was obtained on 2001 March 13 using the CTIO $0.9 \mathrm{~m}$ telescope and the Cassegrain Focus CCD imager ${ }^{1}$. These data were calibrated to the standard system using observations of Landolt standards. The final photometric data set is listed in Table 1. Included is the Two Micron All-Sky Survey (2MASS) JHK photometry of the reference stars, transformed to the homogenized system of Bessell \& Brett (1988) using the transformation equations from Carpenter (2001). Typical error bars on the photometry are $\pm 0.02 \mathrm{mag}$ for the $V$-band measurements, and $\pm 0.03 \mathrm{mag}$ for the optical colors. The 2MASS photometry for the five reference stars has error bars of $\pm 0.02 \mathrm{mag}$. Optical spectroscopy of the reference stars, and a number of MK spectral

\footnotetext{
${ }^{1}$ http://www.ctio.noao.edu/cfccd/cfccd.html
}

type templates, was obtained on 2001 March 9 and 10 using the CTIO $1.5 \mathrm{~m}$ telescope with the Cassegrain Spectrograph ${ }^{2}$ with the $831 \mathrm{l} / \mathrm{mm}$ "G-47" grating and a two arcsecond slit, giving a spectral resolution of $0.56 \AA /$ pix. We estimated spectral types of the reference stars from a comparison of the spectra with those of the MK-templates and present the results in the last column of Table 1 . The visual extinctions of the reference stars (Col. 9 of Table 1) were estimated from a comparison of their spectral types and the photometric data, using the standard relations from Reike \& Lebofsky (1985). Finally, spectroscopic parallaxes were derived using the spectral types and visual extinctions. The results are listed in the last column of Table 2. To determine these values, we used the Hipparcos calibration of the absolute magnitude for main sequence stars by Houk et al. (1997), and that for giant stars tabulated by Drilling \& Landolt (2000). The distinction between main sequence stars and giants is facilitated by (i) the dichotomy of the $M_{V}$-distribution in the Hertzsprung-Russell diagram for stars of spectral type $G$ and later and (ii) the ability of the Bayesian-like astrometric software to identify an object with a true parallax substantially different from the one used as the starting value in the iterative approach. Details of the procedure are given in our previous papers cited above. For the astrometric solution discussed below, we assumed error bars of $\pm 20 \%$ on our spectroscopic parallaxes.

\footnotetext{
${ }^{2}$ http://www.ctio.noao.edu/spectrographs/60spec/ manual/
} 
The reference stars span a wide range in spectral type, and have significant extinctions. Four of the five reference stars turn out to be late-type giants, providing the exceptionally quiet reference frame, which is an essential for the high quality of the parallax derived for V1223 Sgr. Three of them (\#2, \#3, and \#4) have extinctions similar to that of V1223 Sgr which has $A_{V}=$ $0.47 \pm 0.02$ (Bonnet-Bidaud et al. 1982). It is somewhat disquieting that $A_{V}$ does not correlate better with $\pi$. A similar situation, however, was also found in the fields of RR Lyr (Benedict et al. 2002a) and $\delta$ Cep (Benedict et al. 2002b).

\subsection{The astrometric solution}

The data reduction process for deriving a parallax of the target from FGS observations was identical to previous efforts, except for the fact that the astrometer has changed from FGS3 to FGS1R, successfully calibrated by Benedict et al. (2001). With the positions measured by FGS1R, we determine the scale, rotation, and offset plate constants for each observation set relative to an arbitrarily adopted constraint plate. The solved equations are

$$
\begin{aligned}
x^{\prime} & =x+l c(B-V) \\
y^{\prime} & =y+l c(B-V) \\
\xi & =A x^{\prime}+B y^{\prime}+C+R_{\mathrm{x}}\left(x^{2}+y^{\prime 2}\right)-\mu_{x} t_{\mathrm{rel}}-P_{\alpha} \pi_{x} \\
\eta & =D x^{\prime}+E y^{\prime}+F+R_{\mathrm{y}}\left(x^{\prime 2}+y^{\prime 2}\right)-\mu_{y} t_{\mathrm{rel}}-P_{\delta} \pi_{y}
\end{aligned}
$$

where $x$ and $y$ are the rectangular HST coordinates and $l c$ is the derived lateral color correction for color $B-V$ (Benedict et al. 1999). $A, B, D$ and $E$ are a set of scale plate constants, $C$ and $F$ are zero point corrections, $R_{x}$ and $R_{y}$ are radial terms, $\mu_{x}$ and $\mu_{y}$ are the proper motions, $t_{\mathrm{rel}}$ is the time of the observation minus the constraint plate time, $P_{\alpha}$ and $P_{\delta}$ are parallax factors, and $\pi_{x}$ and $\pi_{y}$ are the parallaxes in $x$ and $y$.

As in all our previous astrometric analyses, we employed GaussFit (Jefferys et al. 1987) to minimize $\chi^{2}$. Since we had five high-quality reference star observations at each epoch, we were able to use the eight-parameter model, instead of the six-parameter model used in our previous parallax measurements, which had $A=E$ and $B=-D$. The additional parameters yielded a significantly improved $\chi^{2}$ compared with the simpler model. The input proper motions for V1223 Sgr and the reference stars were taken from the USNO CCD Astrograph Catalog, the "UCAC2" (Zacharias et al. 2004) and are listed in Cols. 3 and 6 of Table 2. The Bayesianlike reduction software models the proper motions and the spectroscopically determined parallaxes of the reference stars as observations with errors to produce an absolute (not relative) parallax for V1223 Sgr. It yields the final proper motions listed in Cols. 4 and 7 of Table 2. The internal consistency of the solution obtained for the V1223 Sgr field is very good. Figure 1 shows the histograms of the residuals for all 220 individual $x$ and $y$ positional measurements of the target and the reference stars. Gaussian fits give standard deviations of 0.88 and 0.90 mas, respectively, which is better than usually obtained for parallax measurements with the HST FGS.

\footnotetext{
${ }^{3}$ ftp://cdarc.u-strasbg.fr/cgi-bin/VizieR, catalogue $\mathrm{I} / 289$.
}
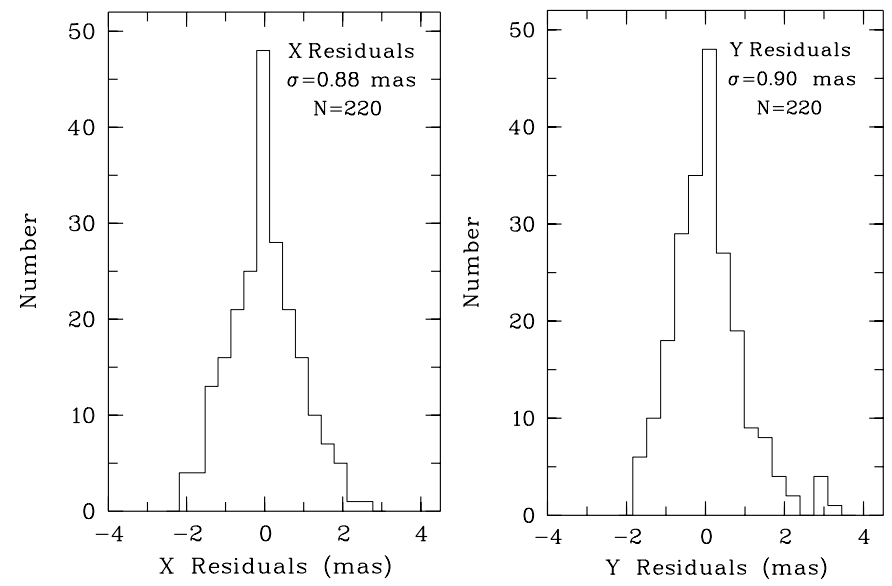

Fig. 1. Histograms of the $x$ and $y$ residuals obtained from modeling V1223 Sgr and its reference frame.

Columns 8 to 11 of Table 2 list the "catalog" positions $\xi$ and $\eta$ and their standard deviations $\sigma_{\xi}$ and $\sigma_{\eta}$ which result from the fit of Eqs. (3) and (4) to the time series of the individual observations. The frequency-weighted mean values of these standard deviations are $\sigma_{\xi}=0.43$ mas and $\sigma_{\eta}=0.46$ mas. Reference star \#3 has standard deviations in both coordinates as low as the other stars, confirming that its input parallax is not grossly in error. Hence, we must presently accept that it is a giant at a distance of $\sim 2.5 \mathrm{kpc}$, although its implied tangential velocity is large.

The final absolute parallax of V1223 Sgr is $\pi=1.96 \pm$ 0.18 mas. The fine precision of this result is directly attributed to the very stable reference frame afforded by the distant giants. The limiting factor in the error budget turns out to be the remaining uncertainty in the proper motions of the reference frame.

\subsection{The distance to V1223 Sgr}

The measured distance to V1223 Sgr is $d=1 / \pi=510_{-43}^{+52} \mathrm{pc}$. As pointed out by Lutz \& Kelker (1973), parallaxes suffer from a systematic error in addition to the observational one in the sense that the most probable true parallax is smaller than the observed absolute parallax because the number of objects per $\pi$-interval increases as $\pi^{-n}$ with $n=4$ for a constant space density. More detailed analyses of the problem including the magnitude and space density distributions of the parent population have been considered, e.g., by Hanson (1979), by Smith (1987), and by Oudmaijer et al. (1998). Here, we have simply assumed that the parent population has an absolute magnitude at the brighter end of the range $0<M_{V}<10$, giving an index of $n=$ 3.0 for the space density (Hanson 1979). The Lutz-Kelker correction is usually quoted as a correction in visual magnitude and, for this choice of parameters, is $\Delta M_{V}=-0.071$. The most probable distance modulus of V1223 Sgr including the Lutz-Kelker correction is $m-M=8.61_{-0.19}^{+0.21}$ and the most probable distance including this correction is $d=527_{-43}^{+54} \mathrm{pc}$. This is the largest distance of a CV so far measured with the HST FGS. 


\section{System parameters and accretion rate of V1223 Sgr}

\subsection{Absolute magnitude}

In its normal high state, V1223 Sgr has a long-term mean magnitude of $B=13.1$ (Garnavich \& Szkody 1988), around which it varies by $\pm 0.5 \mathrm{mag}$. Of this, the orbital and spin modulation accounts for about $\pm 0.3 \mathrm{mag}$. There are also long-term trends and a time span between 1937 and 1951 when it experienced drops to much fainter states (Garnavich \& Szkody 1988). With $B-V \simeq 0$ in the high state (Table 2), $V$ and $B$ are practically the same. From the depth of the $2200 \AA$ feature, Bonnet-Bidaud et al. (1982) derived $E_{B-V}=0.15$ with an error of about 0.01 , which yields a visual absorption $A_{V}=0.47 \pm 0.03$. This value of the absorption, the mean visual magnitude of $V=13.1$, and our new distance modulus give an absolute mean visual magnitude in the normal high state of $M_{V}=4.0 \pm 0.2$. This value has still to be corrected for inclination effects.

\subsection{Secondary star and inclination}

Penning (1985) and Watts et al. (1985) assumed that the mass of the secondary in V1223 Sgr is that of a Roche-lobe filling main sequence star, $M_{2}=0.40 M_{\odot}$. In many CVs, however, the secondaries seem to be expanded over their main sequence radii and have smaller masses than the main-sequence assumption suggests (e.g. Beuermann et al. 1998). We adopt $M_{2}=0.40 M_{\odot}$ as the nominal secondary mass and comment on the effect a mass as low as $0.25 M_{\odot}$ will have on the the results. For a spectral class M4, a secondary in this mass range has a $K$-band magnitude of $K=15.1-15.4$ and contributes $8-11 \%$ of the observed $K$-band flux (Table 1 ).

According to et al. (1985), the radial velocity amplitude of the orbital motion of the white dwarf as derived from the wings of the Balmer emission lines is $K_{1}=56 \pm 13 \mathrm{~km} \mathrm{~s}^{-1}$. Penning (1985), using a superior time resolution, found that the Balmer and He II $\lambda 4686$ radial velocities were modulated, in addition, at the spin period (see his Figs. 9 and 3 and note that the spin modulation is clearly present in his Fig. 3). Penning's $K_{1}$ agrees closely with that of Watts et al., which we adopt here. As noted already by these authors, the implied inclination is small. For $M_{1}=0.93 M_{\odot}$ (see below), we obtain $i=(24 \pm 7)^{\circ}$ with $M_{2}=$ $0.40 M_{\odot}$ and $i=(37 \pm 10)^{\circ}$ with $M_{2}=0.25 M_{\odot}$.

\subsection{X-ray and XUV emission of V1223 Sgr}

\subsubsection{The X-ray spectral analysis of Beardmore et al.}

Our derivation of the white dwarf mass $M_{1}$ and the accretion rate $\dot{M}$ rests on the results of the X-ray spectral analysis of Beardmore et al. (2000). These authors fitted a multitemperature shock model to the ASCA and Ginga X-ray spectra of V1223 Sgr, which included a treatment of the X-albedo and of internal absorption within the source. From this fit, they deduced a shock temperature of $k T_{\mathrm{s}}=43_{-12}^{+13} \mathrm{keV}$ and the emitted spectrum $f_{\text {emi }}(E)$ as a function of photon energy $E$ (called "incident" spectrum by them). Integration of $f_{\mathrm{emi}}(E)$ over all photon energies above the Lyman edge gives the
Table 3. Integrated observed and inferred fluxes for individual wavelength ranges (see text).

\begin{tabular}{llc}
\hline \hline Band & Component & $\begin{array}{c}\text { Flux } \\
\left(\mathrm{erg} \mathrm{cm}^{-2} \mathrm{~s}^{-1}\right)\end{array}$ \\
\hline $0.0136-100 \mathrm{keV}$ & Total emitted X-ray flux & $3.30 \times 10^{-10}$ \\
$>54 \mathrm{eV}$ & Additional XUV source & $<10^{-11}$ \\
$13.6-54 \mathrm{eV}$ & Not observable & $?$ \\
$912-1250 \AA$ & Unobserved FUV & $(2-3) \times 10^{-10}$ \\
$>1250 \AA$ & Observed UV/optical ${ }^{3} / \mathrm{IR}^{4}$ & $7.59 \times 10^{-10}$ \\
\hline
\end{tabular}

${ }^{1}$ Integral of "incident" spectrum of Beardmore et al. (2000); ${ }^{2}$ extrapolated from $\lambda>1250 ;{ }^{3}$ total mean flux from Mouchet $(1983) ;{ }^{4}$ this work.

total flux $F_{\mathrm{x}}=3.30 \times 10^{-10} \mathrm{erg} \mathrm{cm}^{-2} \mathrm{~s}^{-1}$ (Table 3, line 1), which we call "X-ray flux" because only $3 \%$ of it is emitted below $0.1 \mathrm{keV} . F_{\mathrm{x}}$ refers to $k T_{\mathrm{s}}=43 \mathrm{keV}$ and scales, to a first approximation, as $k T_{\mathrm{s}}$. The X-ray luminosity $L_{\mathrm{x}}$ then scales as $k T_{\mathrm{s}}$, too. The emitted spectrum $f_{\mathrm{emi}}(E)$ is based on the observed ASCA spectrum for photon energies $E>0.4 \mathrm{keV}$, but for still lower energies it merely represents an extrapolation of the multi-temperature thermal model spectrum. We demonstrate in the next section, however, that a substantial additional XUV source does not exist in V1223 Sgr.

The observed X-ray luminosity based on the Beardmore et al. (2000) emitted spectrum and our Lutz-Kelker corrected distance from Sect. 2.4, is $L_{\mathrm{x}, 1}=4 \pi d^{2} F_{\mathrm{x}}=(1.11 \pm 0.36) \times$ $10^{34} \mathrm{erg} \mathrm{s}^{-1}$, where the error accounts for the uncertainties in the normalization of the observed X-ray flux, in $k T_{\mathrm{s}}$, and in $d$. We assume that $L_{\mathrm{x}, 1}$ represents the luminosity of one accreting pole and that the second pole is hidden behind the white dwarf. Such a geometry is generally expected if the shock height $h$ is small compared with the white dwarf radius $R_{1}$, as it is in the model of Beardmore et al., and still holds for a finite shock height if the inclination is small and the magnetic axis is more or less aligned with the rotational axis. We account for the second pole by writing $L_{\mathrm{x}}=(1+\beta) L_{\mathrm{x}, 1}$ and use $\beta=1$, below.

\subsubsection{The quest for additional XUV emission}

In magnetic CVs of the polar subtype, a large fraction of the accretion energy is released as soft X-rays and dominates the total X-ray flux, which is used to estimate their accretion rates (Beuermann \& Burwitz 1995). Does a soft X-ray component exist also in the intermediate polar V1223 Sgr?

The XUV luminosity can be estimated by the Zanstra method using the observed line flux $F_{4686}$ of He II $\lambda 4686$ photons with $h v=2.65 \mathrm{eV}$ in erg $\mathrm{cm}^{-2} \mathrm{~s}^{-1}$. The number flux of ionizing photons needed to produce this line is:

$N_{>54}=F_{4686} \frac{\alpha_{\mathrm{B}}}{\varepsilon_{4686}} \frac{4 \pi}{\Omega}$,

where $\Omega$ is the solid angle subtended by the photoionized gas, $\alpha_{\mathrm{B}}$ is the total recombination coefficient of He II for the Baker \& Menzel Case B in $\mathrm{cm}^{3} \mathrm{~s}^{-1}$, and $\varepsilon_{4686}$ the 
emissivity in the $\lambda 4686$ line in $\mathrm{erg} \mathrm{cm}^{3} \mathrm{~s}^{-1}$. For the pure recombination case, $\varepsilon_{4686} / h v_{4686}$ is the effective recombination coefficient for this transition. We use the results of Storey \& Hummer $(1995)^{4}$, which take full account of collisional processes and are tabulated for electron number densities of $N_{\mathrm{e}}=$ $10^{2}-10^{14} \mathrm{~cm}^{-3}$ and a wide range of electron temperatures. They yield $\varepsilon_{4686} /\left(h v_{4686} \alpha_{\mathrm{B}}\right)=0.216(0.140), 0.157(0.125)$, and $0.210(0.156)$ for $N_{\mathrm{e}}=10^{6}, 10^{10}$, and $10^{14} \mathrm{~cm}^{-3}$ and electron temperatures $T_{\mathrm{e}}=10^{4}\left(5 \times 10^{4}\right) \mathrm{K}$, respectively. The particle density in the photoionized region of V1223 Sgr may range from $>10^{14} \mathrm{~cm}^{-3}$ in the disk and in the densest parts of the magnetically confined accretion stream to much lower values elsewhere in the magnetosphere or in the corona of the disk and its bulge, while the temperature is likely between $10^{4}$ and a few times $10^{4} \mathrm{~K}$. The above numbers demonstrate that $\varepsilon_{4686} /\left(h v_{4686} \alpha_{\mathrm{B}}\right)=0.18 \pm 0.05$ is appropriate for a wide range of parameters in the photoionized regions in V1223 Sgr.

Steiner et al. (1981) quote an energy flux of the $\lambda 4686$ line in V1223 Sgr of $9 \times 10^{-14} \mathrm{erg} \mathrm{cm}^{-2} \mathrm{~s}^{-1}$ and other papers suggest values within a factor of two in both directions (Bonnet-Bidaud et al. 1982; Penning 1985; Watts et al. 1985). We adopt the Steiner et al. value which becomes $F_{4686}=$ $1.6 \times 10^{-13} \mathrm{erg} \mathrm{cm}^{-2} \mathrm{~s}^{-1}$ after correction for reddening. Because of the large internal absorption at soft X-ray energies, we use $\Omega \simeq 4 \pi$. This choice of parameters yields $N_{>54} \simeq$ 0.21 photons $\mathrm{cm}^{-2} \mathrm{~s}^{-1}$, with an uncertainty of about a factor of two from the error in $F_{4686}$ and the uncertainties in $\Omega$ and $\alpha_{4686}^{\text {eff }} / \alpha_{\mathrm{B}}$. For comparison, the Beardmore et al. X-ray spectrum $f_{\mathrm{emi}}(E)$ provides 0.11 photons $\mathrm{cm}^{-2} \mathrm{~s}^{-1}$ with energies above $54 \mathrm{eV}$, of which $50 \%$ are below $0.3 \mathrm{keV}$. As argued above, this number probably refers only to the pole facing the observer. The number from both poles would then be 0.22 photons $\mathrm{cm}^{-2} \mathrm{~s}^{-1}$, in agreement with the number needed to produce the observed $\lambda 4686$ flux. We conclude that there is no evidence for an additional component of XUV photons. The conservative limit to the energy flux of a potential XUV source given in line 2 of Table 3 refers to a source which provides the same number of ionizing photons as the X-ray source.

\subsection{An intermediate polar model for V1223 Sgr}

For further discussion of V1223 Sgr, we adopt a simple intermediate polar model in which an accretion disk is broken up by the magnetic field of the primary at an inner radius $r_{\mathrm{i}}>R_{1}$. At $r_{\mathrm{i}}$, matter couples onto the field and falls along the field lines towards both magnetic poles of the white dwarf. We assume that there is no viscous energy release inside $r_{\mathrm{i}}$ and that all gravitationally released energy is radiated in the flow behind the strong shock which forms near the white dwarf surface. This is the usual assumption, which allows to treat the guided motion as a free fall. It neglects the fact that the ionized matter in the funnel has to get rid of the particle momenta perpendicular to the field and does so by radiative losses. Nevertheless, photoabsorption of X-rays in the guided stream and

\footnotetext{
${ }^{4}$ ftp://cdarc.u-strasbg.fr/cgi-bin/VizieR, catalogue VI/64.
}

reemission of the absorbed energy is in line with the model and may cause the funnel to be an intense source of UV/optical radiation. Outside $r_{\mathrm{i}}$, we assume a circularly symmetric steady state disk in which the individual annuli radiate at local effective temperatures $T_{\text {eff }}(r)$ as given by Shakura \& Sunyaev (1973). In addition, we include an inner ring at $r_{\mathrm{i}}$, which mimics the boundary layer and emits the energy released when the matter is braked from the Kepler velocity to the angular velocity of the white dwarf. Following Bonnet-Bidaud et al. (1982) and Mouchet (1983), we use blackbody spectra, which has the advantage of simplicity, but the disadvantage of systematically distorting the spectrum, especially at short wavelengths. For the outer disk, we allow for the possibility of heating by radiation from the central source or from the elevated magnetically guided flow by letting $T_{\text {eff }}(r)$ not drop below a temperature $T_{\min }$. This is obviously a very rough approach, which we justify by its simplicity.

The location of the inner edge of the disk is restricted by two requirements. As a first condition, $r_{\mathrm{i}}$ should not exceed the circularization radius $r_{\text {circ }}$ of matter with the specific angular momentum it carries over from the inner Lagrangian point. No disk can form for $r_{\mathrm{i}}>r_{\text {circ }}$. For $M_{1}=0.93-1.18 M_{\odot}$ and $M_{2}=$ $0.25-0.40 M_{\odot}, r_{\text {circ }}$ falls in the range of $1.06 \times 10^{10}$ to $1.48 \times$ $10^{10} \mathrm{~cm}$. The second condition results from the observation that the white dwarf in V1223 Sgr is being spun down, which indicates that it should rotate close to equilibrium. The equilibrium inner radius of the disk $r_{\mathrm{i}, \mathrm{e}}$ is located inside the corotation radius $r_{\mathrm{co}}=\left(G M_{1} P_{\mathrm{s}}^{2} / 4 \pi^{2}\right)^{1 / 3}=1.23 \times 10^{10} M_{1}^{1 / 3} \mathrm{~cm}$, with $P_{\mathrm{s}}=745.5 \mathrm{~s}$ the spin period of the white dwarf in V1223 Sgr (Osborne et al. 1985). The theory of Ghosh \& Lamb (1979) with later corrections by Wang (1987) predicts $r_{\mathrm{i}, \mathrm{e}} \simeq 0.98 r_{\mathrm{co}}$. This does not account, however, for the synchronization torque between primary and secondary star. Warner (1996) estimates this torque from considerations of the magnetism of the secondary star. For conciseness, we refer to his Eqs. (22)-(24), and note that the magnitude of the effect depends not only on the uncertain magnetic moment of the secondary star, but also on the ratio between inner edge and spherical Alfvén radius, usually taken to be $r_{\mathrm{i}} / r_{\mu \text {,sph }} \simeq 0.52$, and on the poorly known screening of the magnetic moment of the primary by the accretion disk. In our preferred model B (see below), the disk in V1223 Sgr has a rather large central hole and may be quite flimsy. We assume, therefore, that screening is rather weak ( $\delta \simeq 0.2$ in Warner's terminology). For the parameters of V1223 Sgr, we then obtain $r_{\mathrm{i}, \mathrm{e}} \simeq 0.83 r_{\mathrm{co}}=1.0 \times 10^{10} \mathrm{~cm}$, which is smaller than $r_{\text {circ }}$ for the entire range of possible values of $M_{1}$ and $M_{2}$.

This theory also allows to estimate the magnetic moment of the primary and its surface field strength. We use $r_{\mathrm{i}} \simeq 0.52 r_{\mu \text {,sph }}$ with $r_{\mu, \mathrm{sph}}=2^{-3 / 7}\left(G M_{1}\right)^{-1 / 7} \dot{M}^{-2 / 7} \mu^{4 / 7} \mathrm{~cm}$, where $\mu=B_{1} R_{1}^{3}$ is the magnetic moment, $B_{1}$ is the surface field strength of the white dwarf in the orbital plane, and the dipolar magnetic axis is assumed to be aligned with the rotational axis. In the aligned case, the polar field strength is $B_{\mathrm{p}}=2 B_{1}$, while for an inclined magnetic rotator, the factor between $B_{\mathrm{p}}$ and $B_{1}$ is smaller than two. 


\subsection{Derivation of $M_{1}, \dot{M}_{x}$, and $L_{x}$}

The free fall of matter from $r_{\mathrm{i}}$ to $R_{1}$ releases an accretion luminosity $L_{\mathrm{acc}, \mathrm{i}}$ in a shock with temperature $k T_{\mathrm{s}}$

$$
\begin{aligned}
L_{\mathrm{acc}, \mathrm{i}} & =\dot{M} \cdot G M_{1}\left(1 / R_{1}-1 / r_{\mathrm{i}}\right) \\
k T_{\mathrm{s}} & =\left(3 \mu m_{\mathrm{u}} / 8\right) \cdot G M_{1}\left(1 / R_{1}-1 / r_{\mathrm{i}}\right),
\end{aligned}
$$

where $\mu=0.617$ is the molecular weight of all particles for solar composition, $m_{\mathrm{u}}$ is the unit mass, and prompt equilibration between electrons and ions in the shock is assumed. $\mathrm{X}$-ray emission is the major channel of energy release in the post-shock region, but cyclotron emission may also contribute despite the negative results of polarization measurements (Cropper 1986). We represent the cyclotron fraction by a parameter $\alpha$ and write

$L_{\mathrm{acc}, \mathrm{i}}=(1+\alpha) L_{\mathrm{x}}=(1+\alpha)(1+\beta) L_{\mathrm{x}, 1}$.

The quantity $\alpha$, which depends on the field strength and particle density in the post-shock region (e.g. Woelk \& Beuermann 1996), is of the order of 0.1 .

Equation (7) can be solved for $M_{1}$ with $k T_{\mathrm{s}}=43_{-12}^{+13} \mathrm{keV}$ (Beardmore et al. 2000), an adopted value for $r_{\mathrm{i}}$ and a mass radius relation of white dwarfs $R_{1}\left(M_{1}\right)$. We use the relation $R_{1}=\left[1.463-0.885\left(M_{1} / M_{\odot}\right)\right] \times 10^{9} \mathrm{~cm}$, which we found from fitting the radii of $\mathrm{CO}$ white dwarf models by Wood (1995) with a thick hydrogen envelope, an effective temperature of $3 \times 10^{4} \mathrm{~K}$, and masses between 0.80 and $1.20 M_{\odot}$. We use two values of $r_{\mathrm{i}}$, which correspond to the two models considered below, $r_{\mathrm{i}}=8.0 \times 10^{8} \mathrm{~cm}$ and $r_{\mathrm{i}}=1.0 \times 10^{10} \mathrm{~cm}$ (see Sects. 3.6.1 and 3.6.2). The white dwarf masses for these two cases are $M_{1}=1.18 \pm 0.06 M_{\odot}$ and $M_{1}=0.93 \pm 0.12 M_{\odot}$, respectively. A white dwarf mass lower than $\sim 0.8 M_{\odot}$ can be excluded, as noted already by Beardmore et al. (2000).

We derive the accretion rate $\dot{M}_{\mathrm{x}}$ needed to generate the emitted X-ray spectrum $f_{\mathrm{emi}}(E)$ by eliminating $G M_{1}\left(1 / R_{1}-1 / r_{\mathrm{i}}\right)$ from Eq. (6) with help of Eq. (7) and replacing $L_{\mathrm{acc}, \mathrm{i}}$ from Eq. (8). With $L_{\mathrm{x}, 1}$ and $\beta=1$ from Sect. 3.3.1, we then obtain $\dot{M}_{\mathrm{x}}=(1.24 \pm 0.24) \times 10^{17}(1+\alpha) \mathrm{g} \mathrm{s}^{-1}$, where the error depends primarily on the remaining uncertainty in $d$ and the value of $\alpha$. Note that $\dot{M}_{\mathrm{x}}$ is independent of the white dwarf mass. The X-ray luminosity is $L_{\mathrm{x}}=2 L_{\mathrm{x}, 1}=(2.23 \pm 0.71) \times$ $10^{34} \mathrm{erg} \mathrm{s}^{-1}$.

\subsection{Interpretation of the overall UV/optical/IR spectral energy distribution}

In what follows, we interprete the observed UV/optical spectral energy distribution in terms of two simple models and list their parameters in Table 4. Model A is that of a luminous accretion disk, which has its inner edge close to the white dwarf (Bonnet-Bidaud et al. 1982; Mouchet 1983). Model B has the inner edge at $r_{\mathrm{i}}=1.0 \times 10^{10} \mathrm{~cm}$ and requires a different origin for most of the UV emission. We use a distance of $527 \mathrm{pc}$ and a radial velocity amplitude of the white dwarf $K_{1}=56 \mathrm{~km} \mathrm{~s}^{-1}$ (Sect. 3.2). We quote the results in Table 4 for the nominal secondary mass $M_{2}=0.40 M_{\odot}$ and comment on the effect of choosing a lower value of $M_{2}$.

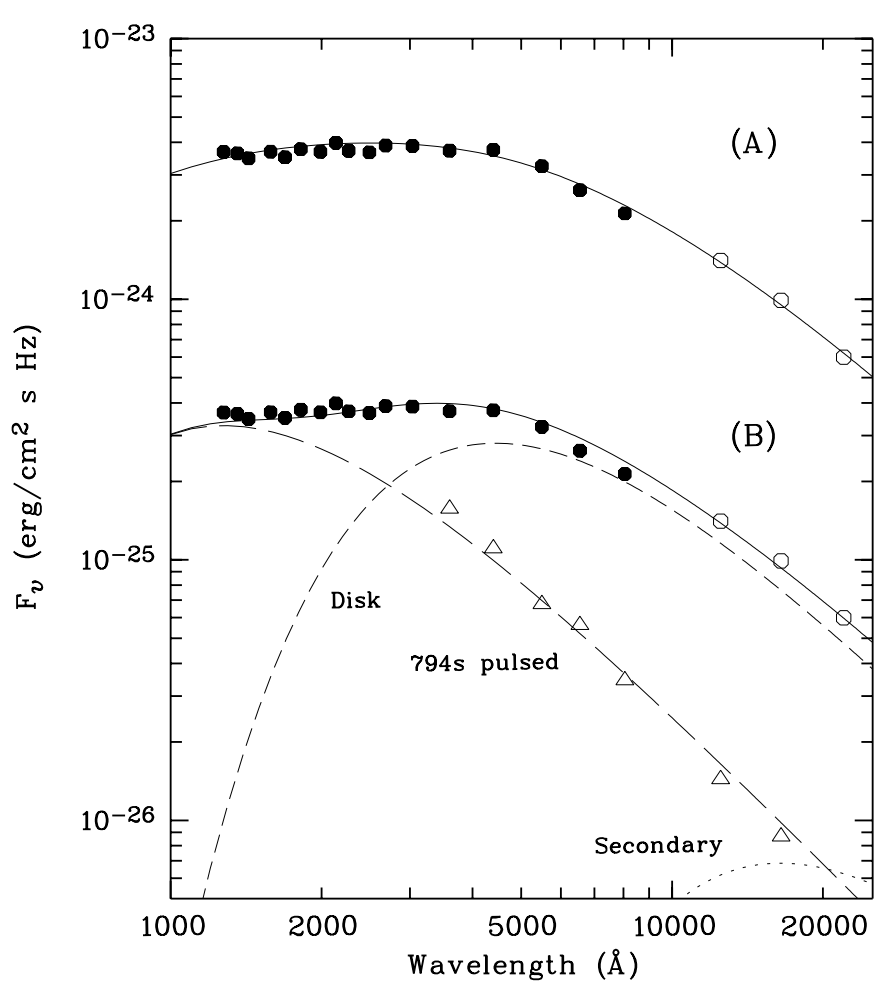

Fig. 2. Overall energy distribution of V1223 Sgr for $\lambda>1000 \AA$ (see text). The data are shown twice with two different model fits. Model A (top data set, fluxes multiplied by 10) is for a luminous steady state accretion disk and model B (bottom data set, fluxes as observed) is for a truncated disk plus reprocessed component.

Figure 2 shows the mean orbital and rotational spectral energy distribution of V1223 Sgr in its normal bright mode. It is based on the IUE spectrophotometry and optical photometry of Bonnet-Bidaud et al. (1982) and Mouchet (1983) (solid circles) and is supplemented by the (non-simultaneous) 2MASS infrared photometry from Table 1 (open circles). The integrated flux for the wavelength range $\lambda>1250 \AA$ is given in Table 3 , line 5, and an estimate of the flux between the Lyman edge and $1250 \AA$ in line 4 .

\subsubsection{Model A: Steady state accretion disk}

The model of a luminous accretion disk (Bonnet-Bidaud et al. 1982; Mouchet 1983) can account for the observed UV/optical/infrared emission. The model requires a high white dwarf mass, an outer disk radius of $90 \%$ of the Roche radius, an inner radius close to the white dwarf, and an accretion rate $\dot{M}_{\mathrm{bb}-\text { disk }}=3.1 \times 10^{17} \mathrm{~g} \mathrm{~s}^{-1}$, which exceeds $\dot{M}_{\mathrm{x}}$ from above. The best fit to the spectral flux distribution is shown in Fig. 2 (upper data set and curve) and the parameters are listed in Table 4 . The spacing between $r_{\mathrm{i}}$ and $R_{1}$ is tailored to yield $k T_{\mathrm{s}}=43 \mathrm{keV}$. The derived accretion rate $\dot{M}_{\text {bb-disk }}$ represents an overestimate because the blackbody disk and its boundary layer at $r_{\mathrm{i}}$ emit $2 / 3$ of the total flux in the Lyman continuum, while typical CV disks have the Lyman jump strongly in absorption. The blackbody disk is a coarse approximation and we quote $\dot{M}_{\mathrm{bb} \text {-disk }}$ in Table 4 in brackets to indicate this uncertainty. If the secondary mass is reduced from 0.40 to $0.25 M_{\odot}$, the blackbody accretion rate 
Table 4. Parameters of disk models A and B. For both models, we assume $M_{2}=0.40 M_{\odot}, K_{1}=56 \mathrm{~km} \mathrm{~s}^{-1}$, and $d=527 \mathrm{pc}$ (see text).

\begin{tabular}{|c|c|c|}
\hline Quantity & Model A & Model B \\
\hline \multicolumn{3}{|l|}{ (1) System parameters: } \\
\hline White dwarf mass $M_{1}\left(M_{\odot}\right)$ & 1.18 & 0.93 \\
\hline White dwarf radius $R_{1}(\mathrm{~cm})$ & $4.17 \times 10^{8}$ & $6.42 \times 10^{8}$ \\
\hline Inclination $i$ & $27.6^{\circ}$ & $24.4^{\circ}$ \\
\hline Separation $a(\mathrm{~cm})$ & $9.21 \times 10^{10}$ & $8.69 \times 10^{10}$ \\
\hline Corotation radius $r_{\mathrm{co}}(\mathrm{cm})$ & $1.30 \times 10^{10}$ & $1.20 \times 10^{10}$ \\
\hline Inner disk radius $r_{\mathrm{i}}(\mathrm{cm})$ & $8.00 \times 10^{8}$ & $1.00 \times 10^{10}$ \\
\hline Outer disk radius $r_{\mathrm{o}}(\mathrm{cm}),\left(0.90 r_{\text {Roche,wd }}\right)$ & $3.48 \times 10^{10}$ & $3.21 \times 10^{10}$ \\
\hline \multicolumn{3}{|l|}{ (2) Temperatures: } \\
\hline Un-irradiated disk: $T_{\max }(\mathrm{K})$ & 58030 & $(6534)$ \\
\hline$T_{\min }(\mathrm{K})$ & 6744 & $(4556)$ \\
\hline Irradiated disk: $\quad T_{\text {irr }}(\mathrm{K})$ & & 11000 \\
\hline "Reprocessed" component (K) & & 40000 \\
\hline \multicolumn{3}{|c|}{ (3) Accretion rate and accretion luminosity for $\alpha=1^{1}$ : } \\
\hline X-ray accretion rate $\dot{M}_{\mathrm{x}}\left(\mathrm{g} \mathrm{s}^{-1}\right)$ & $1.24 \times 10^{17}$ & $1.24 \times 10^{17}$ \\
\hline Blackbody accretion rate $\dot{M}_{\mathrm{bb}-\text { disk }}\left(\mathrm{g} \mathrm{s}^{-1}\right)$ & $\left(3.1 \times 10^{17}\right)$ & \\
\hline Total accretion luminosity $L_{\text {acc }}\left(\mathrm{erg} \mathrm{s}^{-1}\right)$ & & $2.34 \times 10^{34}$ \\
\hline X-ray luminosity $L_{\mathrm{x}}\left(\mathrm{erg} \mathrm{s}^{-1}\right)$ & $2.23 \times 10^{34}$ & $2.23 \times 10^{34}$ \\
\hline \multicolumn{3}{|c|}{ (4) Disk and non-disk/reprocessed fluxes at the Earth ${ }^{2}$ : } \\
\hline Disk $>1250 \AA\left(\mathrm{erg} \mathrm{cm}^{-2} \mathrm{~s}^{-1}\right)$ & $7.61 \times 10^{-10}$ & $2.70 \times 10^{-10}$ \\
\hline Disk $912-1250 \AA\left(\mathrm{erg} \mathrm{cm}^{-2} \mathrm{~s}^{-1}\right)$ & $2.85 \times 10^{-10}$ & $0.02 \times 10^{-10}$ \\
\hline "Reprocessed" > $1250 \AA\left(\mathrm{erg} \mathrm{cm}^{-2} \mathrm{~s}^{-1}\right)$ & & $4.88 \times 10^{-10}$ \\
\hline "Reprocessed" 912-1250 A $\left(\mathrm{erg} \mathrm{cm}^{-2} \mathrm{~s}^{-1}\right)$ & & $2.96 \times 10^{-10}$ \\
\hline \multicolumn{3}{|l|}{ (5) Disk and non-disk/reprocessed luminosities: } \\
\hline Disk > $1250 \AA\left(\mathrm{erg} \mathrm{s}^{-1}\right)$ & $1.23 \times 10^{34}$ & $4.15 \times 10^{33}$ \\
\hline Disk $912-1250 \AA\left(\mathrm{erg} \mathrm{s}^{-1}\right)$ & $4.59 \times 10^{33}$ & $0.03 \times 10^{33}$ \\
\hline "Reprocessed" > $1250 \AA ̊\left(\mathrm{erg} \mathrm{s}^{-1}\right)$ & & $\sim 8.9 \times 10^{33}$ \\
\hline “Reprocessed” 912-1250 А̊ ( $\left.\mathrm{erg} \mathrm{s}^{-1}\right)$ & & $\sim 5.4 \times 10^{33}$ \\
\hline
\end{tabular}

${ }^{1} \dot{M}_{\mathrm{x}}$ and $L_{\mathrm{x}}$ refer to both poles of the white dwarf. $L_{\mathrm{acc}}=G M_{1} \dot{M} / R_{1}$.

${ }^{2}$ Fluxes corrected for interstellar extinction with $A_{\mathrm{v}}=0.47$.

increases to $\dot{M}_{\text {bb-disk }}=5.0 \times 10^{17} \mathrm{~g} \mathrm{~s}^{-1}$, partly because the now larger inclination of $44^{\circ}$ implies a smaller projected area of the disk.

The real problem of the disk model is the location of the inner edge of the disk far inside the corotation radius, which is incompatible with the observed spin down of the white dwarf (van Amerongen et al. 1987; Jablonski \& Steiner 1987). The theory presented above suggests that $r_{\mathrm{i}}$ should be about an order of magnitude larger than assumed in this model. Whatever the remaining uncertainties in the theory, the inner edge of the disk can not be close to the white dwarf.

\subsubsection{Model B: Truncated disk and reverberation of X-rays}

The alternative model $\mathrm{B}$ assumes a disk, which is truncated at $r_{\mathrm{i}}=0.83 r_{\mathrm{co}}=1.0 \times 10^{10} \mathrm{~cm}$. Since the truncated disk is intrinsically faint, we assume that heating by irradiation raises its flux to the observed level, which requires $T_{\text {irr }}=11000 \mathrm{~K}$. The truncated disk is represented in Fig. 2 by an $11000 \mathrm{~K}$ blackbody (short dashed curve). In Table 4, Sect. 4, lines 1 and 2, we list its UV/optical flux. The contribution from the secondary star is minute in comparison (dotted curve for a $3100 \mathrm{~K}$ blackbody adjusted to $K=15.1$ ). Heating the disk to $11000 \mathrm{~K}$ requires that it is moderately inflated. For a point source at the white dwarf, a solid angle of $\Omega \simeq 2$ sr or a half opening angle $\gamma=9^{\circ}$ is needed. If the disk is irradiated by the funnel emission, $\gamma$ could be smaller. In this model, much of the observed UV radiation must be due to the reverberation of X-rays.

That the observed ultraviolet radiation is not entirely of disk origin was indicated already by Mouchet's (1983) observation that the UV flux varied by $23 \%$ over $1.5 \mathrm{~h}$ while the $V$ magnitude stayed constant. Furthermore, Bonnet-Bidaud et al. (1982, see also Welsh \& Martell 1996) showed that the pulsed component has a steeper spectrum than the mean light. When corrected for extinction, it is almost as steep as a Rayleigh-Jeans spectrum, $f_{v} \propto \lambda^{-1.9}$. The wavelength dependence of this component is depicted by the open triangles in Fig. 2, adjusted to $30 \%$ of the total mean flux in the $B$-band. This percentage is at the upper end of the observed range of amplitudes (Steiner et al. 1981; Bonnet-Bidaud et al. 1982; King \& Williams 1983; Warner \& Cropper 1984) and is a reasonable choice if the observed variations in amplitude represent variations in the visibility of the modulation rather than true variations of the pulsed flux. For illustrative purposes, we have adjusted a $40000 \mathrm{~K}$ blackbody to fit these fluxes (Fig. 2, long dashed curve) and the sum of the two components to fit the total observed flux. The "reprocessed" flux is listed in Table 4, Sect. 4, lines 3 and 4. We do not contend that the $794 \mathrm{~s}$ pulsed component extends into the UV as indicated by the $40000 \mathrm{~K}$ blackbody, but suggest that there may exist more than one component of reprocessed light. Combined they may account for much of the UV flux. In view of Penning's (1985) discovery of radial velocity variations at the spin period of the white dwarf, we suspect that a spin-modulated component may still be hidden in the UV. The heated pole cap of the white dwarf is a viable contender. The fact that such a component has not been found at optical wavelengths may be due to a small amplitude of modulation and/or a contrived geometry (Hellier 2003). The sum of truncated disk and "reprocessed" component is seen to fit the data as well as the model of a luminous disk. The present model B has the advantage of being internally consistent with the spin down of the white dwarf and with the X-ray derived accretion rate $\dot{M}_{\mathrm{x}}$. Its parameters depend only weakly on the choice of $M_{2}$.

Is the generation of much of the UV flux by reverberation of X-rays energetically feasible? In order to test this hypothesis, we convert the components of the observed flux $F$ in Table 4 , Sect. 4, to luminosities $L=g d^{2} F$ using appropriate (i.e. non-blackbody) geometry factors $g$. For the "disk" flux we assume a grey limb darkening law, $F_{\mathrm{i}} \propto(1+1.5 \cos i) \cos i$, 
which yields $g=1.85 \pi$ for $i=24.3^{\circ}$. For the "reprocessed" component, $g$ is similar to that of the disk if the heated polar cap is the main source, while an estimated $g \simeq 2.6 \pi$ applies to the toroidal geometry of the magnetically guided flow inside $r_{\mathrm{i}}$. We use an average $g=2.2 \pi$. The resulting luminosities are listed in Table 4, Sect. 5. After corrections for the contributions by the un-illuminated disk and the secondary star, we obtain an upper limit to the reprocessed luminosity $L_{\text {rep, } \max }(>912 \AA)=1.78 \times 10^{34} \mathrm{erg} \mathrm{s}^{-1}=0.80 L_{\mathrm{x}}$. The uncertainty in this number is fairly large because of possible errors in the geometry factors and the remaining uncertainty in the $\mathrm{X}$-ray luminosity. Long-term variability of V1223 Sgr could affect the result, but is probably of minor importance because the AAVSO records (Mattei 2003) show V1223 Sgr at $V=13.1$ at the time of the ASCA X-ray observations, in agreement with its long-term mean magnitude. The load on the X-ray source is reduced if part of the optical emission is of cyclotron origin, represented by the quantity $\alpha$ introduced in Sect. 3.4, which depends on the field strength and the pre-shock mass flow density $\dot{m}$ (in $\mathrm{g} \mathrm{cm}^{-2} \mathrm{~s}^{-1}$ ). From $\dot{M}_{\mathrm{x}}$ and an estimate of the area of the ring-shaped accretion region on the white dwarf we expect $\dot{m} \simeq 3-10 \mathrm{~g} \mathrm{~cm}^{-2} \mathrm{~s}^{-1}$. For $B \simeq 10-15 \mathrm{MG}$, we then estimate $\alpha \simeq 0.05-0.15$ using the radiation-hydrodynamic calculations of Woelk \& Beuermann (1996, their Fig. 9, bottom panel, lower curve). For $\alpha \simeq 0.10, L_{\mathrm{rep}} / L_{\mathrm{x}}$ drops to a comfortable 0.69 , which can easily be accounted for by X-ray heating of the pole cap of the white dwarf and reprocessing in the funnel, in the accretion disk, and the irradiated face of the secondary star (see Beardmore et al. 2000 for an analysis of the energy-dependent X-ray albedo from the white dwarf). For $\alpha=0.1 \pm 0.05$, the accretion rate and the total accretion luminosity increase over the values quoted in Sect. 3.5 and in Table 4 to $\dot{M}=(1+\alpha) \dot{M}_{\mathrm{x}}=(1.36 \pm 0.27) \times 10^{17} \mathrm{~g} \mathrm{~s}^{-1}$ and $L_{\mathrm{acc}}=1.049(1+\alpha) L_{\mathrm{x}}=(2.57 \pm 0.83) \times 10^{34} \mathrm{erg} \mathrm{s}^{-1}$, where the errors are from Sect. 3.5 and the numerical factor in the last relation accounts for the energy released outside $r_{\mathrm{i}}$. Since the field estimate given below suggests that some cyclotron emission should be present, we accept the latter values as our best estimates.

\subsection{Magnetic moment and field strength of the white dwarf}

The different sizes of the central holes in the accretion disks imply different surface field strengths $B_{1}$ of the white dwarfs in models $\mathrm{A}$ and $\mathrm{B}$. A rough estimate of $B_{1}$ may be obtained by equating $r_{\mathrm{i}}$ with $0.52 r_{\mu \text {,sph }}$ (see above). For model B with $r_{\mathrm{i}} \simeq 1.0 \times 10^{10} \mathrm{~cm}, \mu=2.06 \times 10^{33} \mathrm{G} \mathrm{cm}^{3}$, and for $r_{1}=$ $6.42 \times 10^{8} \mathrm{~cm}$ (Table 4$), B_{1}=8 \mathrm{MG}$. Depending on the obliquity of the dipole, the polar field strength is in the range of $B_{\mathrm{p}}=10-16 \mathrm{MG}$. The small inner hole of the disk with $r_{\mathrm{i}}=8.0 \times 10^{8} \mathrm{~cm}$ in model A implies a much smaller surface field strength of about 0.5 MG. Our favored model B places V1223 Sgr at the lower range of field strengths observed in polars. Since low-field polars are characterized by weak soft X-ray emission, the lack of a strong soft X-ray source in V1223 Sgr is not surprising.

\section{Conclusion}

We have presented an accurate parallax of V1223 Sgr which allows us to derive the luminosities in the different wavelength bands. Based on this result, we have tested the hypothesis that much of the UV/optical emission is produced by the reverberation of X-rays and not by the release of gravitational energy in a luminous disk. Our analysis is based on the assumption that we see only the X-rays from one pole. This assumption and the high X-ray temperature reported by Beardmore et al. (2000) lead to a total X-ray luminosity $L_{\mathrm{x}}=(2.2 \pm 0.7) \times 10^{34}$, which is much higher than previously thought and can power a large fraction of the observed UV/optical radiation by the reprocessing of X-rays. Interestingly, this is possible without the presence of a substantial source of XUV radiation. Likely reprocessing sites are the pole cap of the white dwarf, the magnetically guided accretion flow, the disk, and the irradiated face of the secondary star. While the latter two sites may be responsible for the observed flux modulated at the sideband frequency (Steiner et al. 1981), reprocessing in the former locations may produce a so far undiscovered component, which is photometrically modulated at the spin period of the white dwarf. The accretion rate derived from the X-ray luminosity and X-ray temperature is $\dot{M}=(1.4 \pm 0.3) \times 10^{17} \mathrm{~g} \mathrm{~s}^{-1}$, which includes a small correction for the contribution by cyclotron emission. This result is independent of the white dwarf mass.

The observed UV/optical/IR spectral energy distribution can equally well be fitted by a truncated disk plus reprocessed component (model B) and by a luminous disk (model A). The observed spin-down of the white dwarf (Jablonski \& Steiner 1987; van Amerongen et al. 1987), however, requires that the the inner edge of the disk is not too far inside the corotation radius, a condition which is met only by the truncated-disk model B.

Strong internal absorption and the reprocessing of a major fraction of the emitted X-rays is a general feature observed also in other intermediate polars, some of which are even more strongly internally absorbed than V1223 Sgr (e.g. Norton \& Watson 1989). Simultaneous X-ray/UV studies could shed light on the physical processes acting in these systems. It is surprising that no such study is yet available.

Acknowledgements. One of (K.B.) thanks Andrew Beardmore for providing his model fits to the X-ray spectrum of V1223 Sgr and Coel Hellier for a stimulating discussion on the physics of intermediate polars. We thank the referee John Thorstensen for pointing out errors in Table 2 and for very helpful comments which led to an improved presentation. This research was supported in Germany by DLR/BMFT grant 50 OR 99031 . In the UK, BTG was supported by a PPARC Advanced Fellowship. In the Unites States, partial support for TEH, BEM, and GFB for proposal \#9230 was provided by NASA through a grant from the Space Telescope Science Institute, which is operated by the Association of Universities for Research in Astronomy, Inc., under NASA contract NAS 5-26555. This research has made use of the NASA/ IPAC Infrared Science Archive, which is operated by the Jet Propulsion Laboratory, California Institute of Technology, under contract with the National Aeronautics and Space Administration. This publication also makes use of data products from the Two Micron All Sky Survey, which is a joint project of the University of Massachusetts and the Infrared Processing and 
Analysis Center/California Institute of Technology, funded by the National Aeronautics and Space Administration and the National Science Foundation. We acknowledge with thanks the variable star observations from the AAVSO International Database contributed by observers worldwide and used in this research.

\section{References}

Beardmore, A. P., Osborne, J. P., \& Hellier, C. 2000, MNRAS, 315, 307

Benedict, G. F., McArthur, B. E., Chappell, D. W., et al. 1999, AJ, 118,1086

Benedict, G. F., McArthur, B. E., Nelan, E. P., \& Jefferys, W. H. 2001, A\&AS, 198, 63.01

Benedict, G. F., McArthur, B. E., Fredrick, L. W., et al. 2002a, AJ, 123,473

Benedict, G. F., McArthur, B. E., Fredrick, L. W., et al. 2002b, AJ, 124,1695

Bessell, M. S., \& Brett, J. M. 1988, PASP, 100, 1134

Beuermann, K., \& Burwitz, V. 1995, ASP Conf. Ser., 85, 99

Beuermann, K., Baraffe, I., Kolb, U., \& Weichhold, M. 1998, A\&A, 339,518

Beuermann, K., Harrison, T. E., McArthur, B. E., Benedict, G. F., \& Gänsicke, B. T. 2003, A\&A, 412, 821

Bonnet-Bidaud, J. M., Mouchet, M., \& Motch, C. 1982, A\&A, 112, 355

Carpenter, J. M. 2001, AJ, 121, 2851

Cropper, M. 1986, MNRAS, 222, 225

Drilling, J. S., \& Landolt, A. U. 2000, in Astrophysical Quantities, ed. A. Cox (New York: Springer-Verlag), 381

Garnavich, P., \& Szkody, P. 1988, PASP, 100, 1522

Ghosh, P., \& Lamb, F. K. 1979, ApJ, 234, 296

Hanson, R. B. 1979, MNRAS, 186, 875

Harrison, T. E., McNamara, B. J., Szkody, P., et al. 1999, ApJ, 515, L93

Harrison, T. E., McNamara, B. J., Szkody, P., \& Gilliland, R. 2000, AJ, 120, 2649

Harrison, T. E., Johnson, J. J., McArthur, B. E., et al. 2003, AJ, 127, 460
Hellier, C. 2003, private communication

Houk, N., Swift, C. M., Murray, C. A., Penston, M. J., \& Binney, J. J. 1997, in Hipparcos - Venice, ed. M. Perryman (Noordwijk: ESA), 279

Jablonski, F., \& Steiner, J. E. 1987, ApJ, 323, 672

Jefferys, W., Fitzpatrick, J., \& McArthur, B. 1987, Celest. Mech., 41, 39

King, A. R., \& Williams, G. A. 1983, MNRAS, 205, 57P

Lutz, T. E., \& Kelker, D. H. 1973, PASP, 85, 573

Mattei, J. A. 2003, Observations from the AAVSO International Database, private communication

McArthur, B. E., Benedict, G. F., Lee, J., et al. 1999, ApJ, 520, L59

McArthur, B. E., Benedict, G. F., Lee, J., et al. 2001, ApJ, 560, 907

Mouchet, M. 1983, IAU Coll., ed. M. Livio, \& G. Shaviv, 72, 173

Norton, A. J., \& Watson, M. G. 1989, MNRAS, 237, 853

Oudmaijer, R. D., Groenewegen, M. A. T., \& Schrijver, H. 1998, A\&A, 294, L41

Osborne, J., Rosen, R., Mason, K. O., \& Beuermann, K. 1985, Sp. Sc. Rev., 40, 143

Penning, W. R. 1985, ApJ, 289, 300

Reike, G. H., \& Lebofsky, M. J. 1985, ApJ, 288, 618

Shakura, N. I., \& Sunyaev, R. A. 1973, A\&A, 24, 337

Smith, H. 1987, A\&A, 188, 233

Steiner, J. E., Schwartz, D. A., Jablonski, F. J., et al. 1981, ApJ, 249, L21

Storey, P. J., \& Hummer, D. G. 1995, MNRAS, 272, 41

van Amerongen, S., Augusteijn, T., \& van Paradijs, J. 1987, MNRAS, 228, 377

Wang, Y.-M. 1987, A\&A, 183, 257

Warner, B. 1996, Astrophys. Space Sci., 241, 263

Warner, B., \& Cropper, M. 1984, MNRAS, 206, 261

Watts, D. J., Giles, A. B., Greenhill, J. G., et al. 1985, MNRAS, 215, 83

Welsh, W. F., \& Martell, P. J. 1996, MNRAS, 282, 739

Woelk, U., \& Beuermann, K. 1996, A\&A, 306, 232

Wood, M. 1995, in Lecture Notes in Physics, ed. D. Koester, \& K. Werner, LNP, 443, 41

Zacharias, N., Urban, S. E., Zacharias, M. I., et al. 2004, AJ, in press 